\title{
Efficacy of Early Yakson Touch and Kinaesthetic Stimulation (Yakin) on the Development of High Risk Neonates: A Randomized Controlled Trial Protocol
}

\section{Parashar $\mathbf{P}^{1}$, Samuel AJ², Aranha VP³}

\begin{abstract}
Introduction: About 15 million babies are born preterm annually, with every year almost 1 million dying from complications and survivors prone to lifetime disability. Yakson touch and kinaesthetic stimulation techniques have proven effective in minimizing the complications and disability of preterm delivery, but presently there is no standardized protocol for their application. The aim is to investigate the efficacy of Yakson touch in combination with kinaesthetic stimulation (YAKIN) on the motor and behavioural development of neonates at high risk of development delay in comparison to conventional handling. Material and Methods: Neonates will be recruited via parental consent from a recognized tertiary care teaching hospital for this two-group pretest-posttest design of randomized controlled trial (RCT), with allocation into either a control group $(n=14)$ or experimental group $(n=14)$ by block randomization method. The experimental group will receive Yakson touch and kinaesthetic stimulation, whilst the control group will receive conventional handling inclusive of developmental positioning and Kangaroo mother care. The duration of intervention will be five out of seven days. Neonatal behavioural assessment scale (NBAS) will be performed at baseline and then on the 7th day to ascertain the intervention effectiveness. Results: Data analysis will be done by Wilcoxon signed rank test/paired t-test and Mann Whitney $\mathrm{U}$ test/independent t-test will be done to compare the pre and post intervention score of NBAS within and between groups. The level of significance will be set as $p<0.05$. Conclusion: The present study may provide valuable information about early intervention to the neonatal physiotherapist, intensivist, nursing staff and parents of neonates.
\end{abstract}

Key words: Development, Low birth weight, Preterm, neonates, Yakson.

\section{Introduction}

Aborn eonate is a baby under 28 days or 4 weeks of age whilst a neonate born in less than 37 weeks of gestation is a preterm neonate ${ }^{1}$ and is considered a high-risk neonate as they are prone to delayed growth and development, mental and physical disability, life time disability
${ }^{1}$ Preeti Parashar, Post Graduate Student, ${ }^{2}$ Asir John Samuel, Orchid ID: http://orcid.org/00000003-1747-0415, Associate Professor, ${ }^{3}$ Vencita Priyanka Aranha, Assistant Professor from the Department of Paediatrics and Neonatal Physiotherapy, Maharishi Markandeshwar Institute of physiotherapy and Rehabilitation, Maharishi Markandeshwar University, Mullana133 207, Ambala District, Haryana, India.

\section{Address for correspondence \\ Asir John Samuel, Associate Professor, Maharishi Markandeshwar Institute of Physiotherapy and Rehabilitation, Maharishi Markandeshwar University, Mullana, Ambala District, Haryana, India Tel No; +91 8059930222 \\ E-mail: asirjohnsamuel@mmumullana.org}

Acknowledgements: Sincere thanks to Dr. Kanimozhi Narkeesh, Professor and Principal, MMIPR, Maharishi Markandeshwar University, Haryana, India and Dr. Narkeesh Arumugam, Professor, Faculty of Medicine, Punjabi University, Patiala, Punjab, India, towards the successful completion of this study.

Funding: Nil

\section{Conflict of Interest: None}

Permission from IRB: This study is approved by Maharishi Markandeshwar University -Institution Ethics Committee (IEC/ MMU/2016/751) and the trial is registered under ClinicalTrials.gov, NCT02815904

\section{How to cite}

Parashar P, Samuel AJ, Aranha VP. Efficacy of Early Yakson Touch and Kinaesthetic Stimulation (Yakin) on the Development of High Risk Neonates: A Randomized Controlled Trial Protocol. J Nepal Paediatr Soc 2017;37(2):184190.

\section{doi:http://dx.doi.org/10.3126/jnps.v37i2.16724}

This work is licensed under a Creative Commons Attribution 3.0 License. 
such as learning disability, visual or hearing impairments, or even increased risk of death ${ }^{2,3,4}$. The preterm new born moves to smaller degrees as compared to full term newborns ${ }^{5}$. In high risk neonates there are increased chances of appearance of development impairments at later stage of development ${ }^{6,7,8}$. Stimulation given at an early stage to neonates can alter the growth of the brain cells, improve adaptive behaviour, and thus age appropriate development is likely to be achieved ${ }^{6}$.

Yakson is a Korean therapeutic touch given to neonates and infants by caressing their abdomen with one hand while the other hand is placed on the back of the neonate to relieve their pain and to calm them down. It was traditionally used by Koreans by caressing the aching body part of their children with a belief that it would relieve their pain ${ }^{2}$. Yakson is one of the most effective and safe methods of tactile stimulation for preterm neonates ${ }^{2,8}$. Tactile and kinaesthetic stimulation techniques (such as massage and rhythmic passive limb movements) have proven to be effective on preterm neonate's development but there is no standardized protocol for these interventions. Therefore there is need to describe the methodology as well as the effects accurately ${ }^{9}$. The role of physiotherapy interventions in treatment of high risk neonates has not been established ${ }^{10,11,12}$. The high risk neonates are prone to developmental delays with early intervention being the most effective for them owing to the plasticity of brain in early infancy ${ }^{5,6}$.

Pepinoa and Mezzacappa concluded that tactile kinaesthetic stimulation is a promising technique potentially associated with faster weight gain, shorter hospital stay, and better behavior, among others, but there is no standard protocol for its application ${ }^{9}$. Aliabadi and Askary examined the effects of tactile-kinaesthetic stimulation on the physical and behavioural development of infants with low birth weight (LBW) (>1500g and $<2499 \mathrm{~g}$ ). They found that tactile-kinesthetic stimulation did not have any adverse physiological effects on the physiological parameters and helped in improvement of adaptive behaviour of the LBW infants ${ }^{6}$. Kachoosangy and Aliabadi stated that tactile kinaesthetic stimulation is effective in improving the motor behaviour, but suggested that there is need for studies in other aspects of behaviour in LBW infants ${ }^{13}$.

Ferreira and Bergamasco concluded that tactile and kinaesthetic stimulation had a positive effect on adjustment and self-regulation behaviour of preterm neonates ${ }^{14}$. They implied the need for studies that will focus on the effects of these interventions. Eshghi et al concluded that Yakson therapeutic touch increased the sleep scores and thus significantly affected the behavioural response of premature infants ${ }^{8}$. Bijari et al concluded that Yakson and gentle human touch increased sleep states of the infants and can decreased stress and energy consumption in infants in neonatal intensive care unit $(\mathrm{NICU})^{2}$. Since there is lack of significant evidence for combined use of Yakson and kinaesthetic stimulation (YAKIN) to improve development of high risk neonates ${ }^{15}$,

The present study is aimed at determining the combined effects of tactile stimulation in the form of Yakson touch and kinaesthetic stimulation in the form of passive movements on LBW neonates (<2500gm) and preterm neonates ( $<37$ weeks) through a RCT.

\section{Material and Methods}

The study design will be a single-blinded RCT of two-group pretest-post test design ${ }^{16}$. Ethical clearance has been obtained from the Research and Student Project Committee of Maharishi Markandeshwar Institute Physiotherapy and Rehabilitation, Mullana, Haryana and Institutional Ethical Committee (IEC) of Maharishi Markandeshwar University (IEC/MMU/2016/751). The study will be performed in accordance with the guidelines laid out by the Indian Council of Medical Research ICMR (2006) (http://www.icmr.nic.in/ ethical_guidelines.pdf) and the Helsinki Declaration (Revised 2013) (http://www.wma.net/en/20activities/10 ethics/10helsinki/DoH-Oct2013-JAMA.pdf). The trial is registered under ClinicalTrials.gov, NCT02815904. This single centre study will be conducted within the NICU, Department of Paediatrics of a recognized tertiary care teaching hospital. Parents of infants born prematurely and with LBW will be approached for consent prior to their child being randomly allocated into one of two groups - an intervention group or the 'usual care' control group. Parents of all the potential participants will receive a written explanation of the trial and given written informed consent forms to be signed prior to their child being involved in the trial. Once parental consent is obtained, samples will be recruited via simple random sampling method using a random number generator, an application provided by the Statistical Package for Social Sciences (SPSS version 16. SPSS Inc., Chicago, IL, USA.)

Participant's recruitment, interventions and outcomes: Recruitment for this study will be undertaken for a total duration of 1 year between June, 2016 and May, 2017. Neonates of either gender with birth weight less than 2500 grams and born before 37 weeks of gestation will be recruited into the study in the first week of their life. Medically unstable neonates requiring lifesaving interventions such as assisted ventilation, cardiac support medicines etc. or neonates with communicable 
disease or congenital anomaly or skin infection will be excluded from the study. After randomization each neonate will receive their allocated interventions once a day for 1 week ( 5 days a week) for 25 minutes a day after the random allocation.

Yakson touch and kinaesthetic stimulation will constitute the independent variables while the dependent variable will be development of neonates as per the scores of NBAS. Outcomes of the study will be measured by the qualified primary researcher in terms of the NBAS at baseline and end of 1 week intervention. The primary researcher had undertaken sufficient training in completing the NBAS on neonates before study commencement. The detailed schematic diagram of the study protocol is displayed in Figure 1.

Sample size: Sample size has been estimated on the basis of unpublished pilot study data. The formula for sample size is comparison of means of two-sample parallel design study ${ }^{17}, n=2[(Z \alpha+Z \beta) s / d]^{2}$ (where $Z \alpha=$ level of significance, $Z \beta=$ power of study, $s=$ pooled SD of the two samples, $d=$ minimal clinically important difference (MCID) for NBAS total score based on our pilot study. Za is set as 1.98 , as a will be assumed to be 0.05 to greatly minimize type 1 error. $Z \beta$ is 0.84 and thereby maintaining the power of the study at $80 \%$. From the pilot study, the following have been obtained $\mathrm{s}=18.9$ and $d=20.9$. Thus a sample size of 14 in each group considering $10 \%$ dropout rate, has been determined.

Assignment of intervention: Neonates meeting the inclusion criteria for the study will be identified and following parental consent will be randomly allocated to one of two groups: $A$ - the experimental group $(n=14)$, or $B$ - the control group $(n=14)$, Figure 1. By the block randomization method, the neonates will be randomly allocated using sequentially numbered, opaque sealed, envelopes. The parents of the neonates will be instructed not to reveal the allocated groups of their baby until the study begins. An independent associate investigator who is not involved in the study intervention delivery will generate the allocation sequence, enrol the participants, and assign participants to their allocated group. As the primary researcher is a post-graduate student and the study will be undertaken as a part of her curriculum, blinding during recruitment, intervention and data collection phases will not be possible. Data analysis will be performed by a blinded independent researcher.

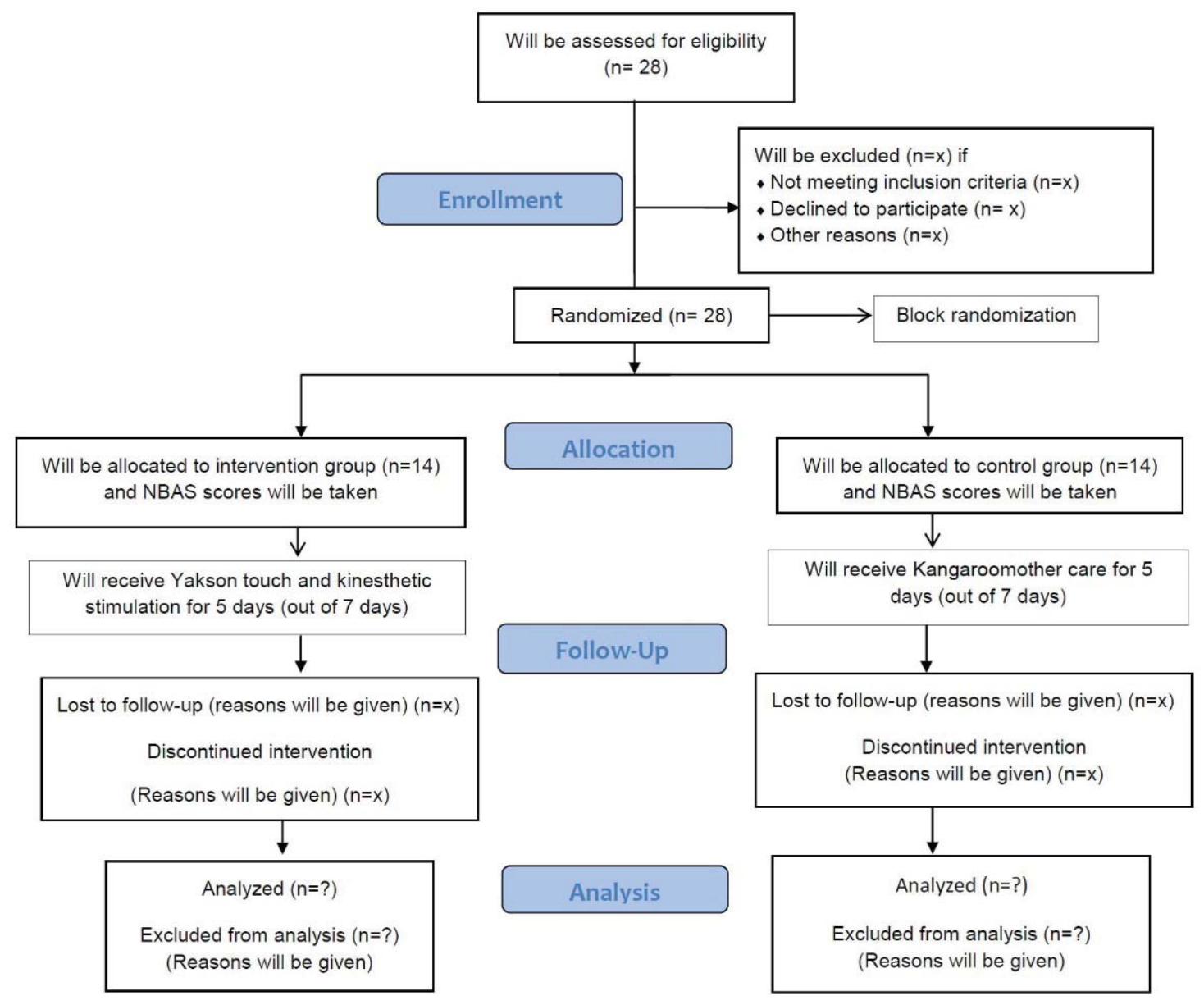

Fig 1: Schematic flow chart for the study protocol (YAKIN) 
Experimental group treatment: Applying Yakson touch (stepwise procedure, see Figure 2)

i. Universal precautions to maintain sterile conditions will be taken.

ii. Then the therapist will warm both hands with a radiant warmer so that the temperature of the palms reaches between $93 \mathrm{~F}$ and 94F (33.9$34.4^{\circ} \mathrm{C}$ ). Digital thermometer will be used to check the temperature of palms. By energy loss or decay mechanism, it is not uncommon to observe decay or loss of heat from palm. Heat loss is inevitable during the application of Yakson touch through palms. But as the radiant warmer will be in on state when executing the intervention, the energy loss would be minimal.

iii. The therapist will relax arms and shoulder muscles for 1 minute and will do deep breathing to accumulate Ki energy on the palms.

iv. The therapist will apply Yakson to the neonate with the neonate being in supine position. Yakson will be given for 15 minutes which will be divided into resting the hands, caressing gently, and again resting the hands, each of which will last for 5 minutes. Throughout the application of Yakson,

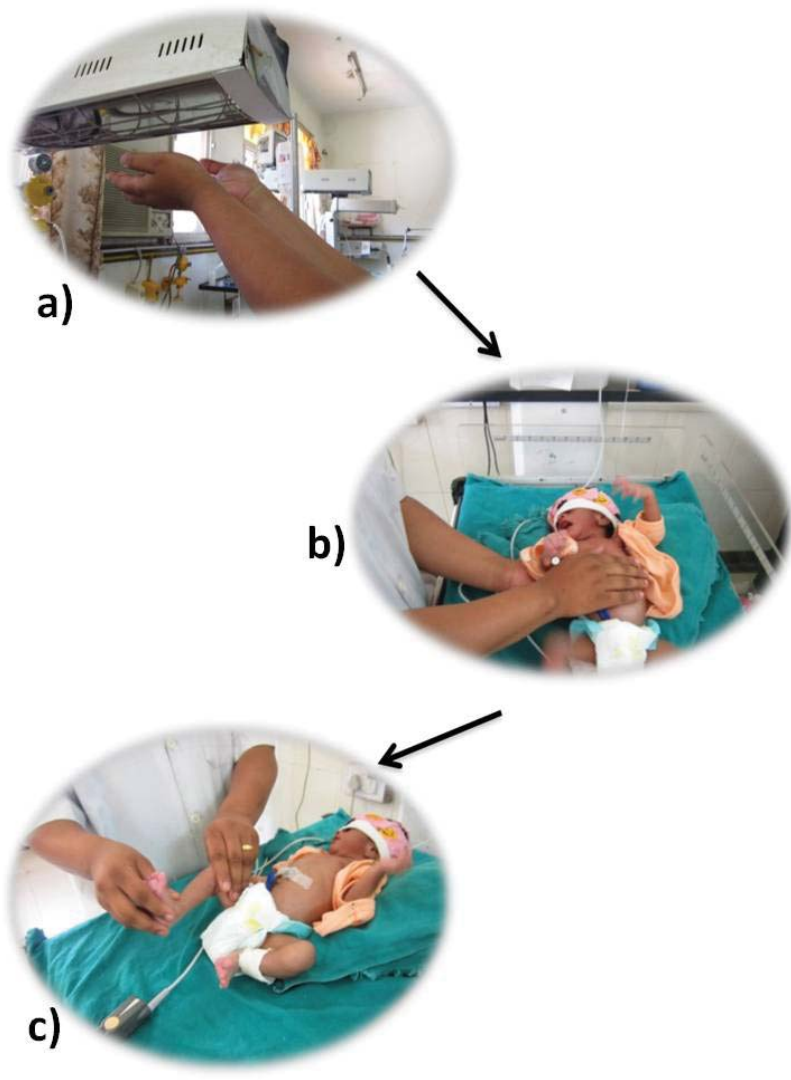

Fig 2: Stepwise procedure for applying Yakson touch. a) Warming both hands with a radiant warmer; b) Applying Yakson on the neonate; c) Applying kinaesthetic stimulation to the neonate. fingers and palm of the therapist will be in constant contact and the contact will be such that there is no pressure on the infant.

v. Resting the hands for 5 minutes- while one hand is resting on the chest and abdomen of the neonate and other hand is supporting the back, the therapist will concentrate on resting hands assuming that a healthy Ki energy is passing to the neonate. During this, the therapist will do slow breathing to remain relaxed.

vi. Caressing gently for 5 minutes-with hand in same position, the therapist will caress repeatedly for 5 minutes. Therapist will caress for 1 minute, rest for 30 seconds, caress for 1 minute, rest for 30 seconds and caress for 2 minutes. The therapist will caress the abdomen and chest of the infant in clockwise direction in a diameter of 1 centimetre in circular motion every 10 seconds

vii. Resting the hands for 5 minutes- the therapist will rest the hands as previously described.

Kinaesthetic stimulation: Immediately following the Yakson, for giving kinaesthetic stimulation the neonate will remain in supine position. There will be six passive flexion and extension movements per limb. Each of the movement will each last for approximately 10 seconds. The movements will be performed in the following order: right arm, left arm, right leg, left leg and both legs simultaneously involving full range of motion in major joints like shoulder and hip joints.

Control group treatment: The neonates in the control group will receive Kangaroo mother care18 for one hour a day and developmental positioning (positioning of preterm infants for optimal physiological development) for 20 minutes per hour for 5 days.

Data collection: The NBAS will be used to monitor the effects of YAKIN on the development of high risk neonates. Baseline reading of NBAS will be recorded for all enrolled neonates before the beginning of intervention. The NBAS is an instrument designed to assess full-term babies from 37-48 weeks' gestation with excellent test-retest reliability; it has been used in over 700 studies worldwide, as a systematic way of observing the individual baby's responses to moderately aversive (e.g. reflexes, motor tone) and non-aversive (e.g. tracking, turning to sound) stimulation. The NBAS contains 28 behavioural items, 18 reflex items, and 7 supplementary items for fragile or high-risk infants. It is a neurobehavioral screening tool, and will identify gross abnormalities or asymmetries. All items will be administered when the baby is in the appropriate behavioural state (states 1-6, from deep sleep to crying), and there is a sequence to follow. The NBAS is 
a structured examination to demonstrate both negative and positive characteristics of infant's behavior. The motor system in NBAS includes the general tone, motor maturity, pull-to-sit, defensive, and activity level ${ }^{19}$. There are six main clusters/packages scored 1-9 (28 items), seven supplementary items scored $1-9$, and 18 reflex / motor items scored $0-3^{20}$. The scoresheet includes 27 behavioral items, each of which is scored on a nine point scale, each of which is scored on a three point scale $^{21}$. Time taken in administration is $20-30$ minutes. It is a naturalistic observation, elicited items and handling required administered according to infant's state ${ }^{20}$.

The sleep states are - (i) Deep sleep with regular breathing, eyes closed, (ii) Light sleep with eyes closed; rapid eye movements can be observed under closed lids; low activity level. Awake States are (iii) Drowsy or semi-dozing; eyes may be open or closed, eyelids fluttering; activity level variable.(iv) Alert, with bright look, Minimal motor activity (v) Eyes open; considerable motor activity, (vi) Crying ${ }^{21}$. The domains are habituation, social interactive, motor system, state organization, state regulation, autonomic system, supplementary system and reflexes ${ }^{19}$.

To minimize dropouts and to facilitate participant retention, parent of the neonates will be assured $30 \%$ fee concession towards paediatric physiotherapy consultation. They will be reminded of the physiotherapy visits over Short Messaging Services (SMS) and if their visits were delayed by two days, then telephonic followup would be done.

To ensure confidentiality each participant will be assigned a serial code for data collection and analysis; for example, $\mathrm{C} 1$ for control group first participants and E1 for the first participant in the experimental group. The primary investigator will be the only person to hold the key to the code linking participant details and their data. The data will be entered in the Microsoft@ Office Excel 2010 using encrypted user password. Moreover, the data will be stored in desktop with no internet connection and sealed Universal Serial Bus (USB) port. This will prevent unauthorized data access and data theft. The final data will also be stored on a non-rewritable Compact Disc Read-only Memory (CD-ROM), again with encrypted user password. All the encrypt user password will be shared between the primary researcher and the data analyst only. Data will be archived for future reference without names or any other features which can reveal the participant identities.

Data and Safety Monitoring Committee: Data collected will be overseen by the Data and Safety Monitoring Committee (DSMC), which is headed by the Chairman of the Student Project Committee. It is independent from the primary researcher and has its role in monitoring the outcome of the intervention and to alert the researcher if any adverse results such as more than $20 \%$ declining tendency from NBAS baseline score occurs. Interim analyses will be performed, when atleast $50 \%$ of neonates have been randomized, to check whether the effect of intervention is proceeding as expected or not and to document other unintended effects of trial interventions. If any pre-determined adverse events occur (such as SPO2 < 89, $100>\mathrm{HR}$ $>200$ beats/min, etc.) then cessation of study protocol guidelines will be strictly adhered to. It has been fixed that DSMC will audit the trial conduct once in 15 days by an independent statistician. The date and time of the audit will not be disclosed to the primary researcher.

The data will be collected, entered and analysed by the primary researcher. Either the Wilcoxon signed rank test or a paired t-test will be used to compare the pre and post intervention score of the NBAS, depending on whether the data are normally distributed or not. Similarly, a Mann Whitney $U$ test or an independent t-test will be used to compare the NBAS scores of experimental group and control group. Data will be analysed using the statistical software (SPSS version 16. SPSS Inc., Chicago, IL, USA.). Level of significance will be set as 0.05 . Missing data due to unavoidable causes will be managed by intention-to-treat (ITT) analysis ${ }^{22,23}$.

\section{Discussion}

A preterm neonate in NICU is deprived of what a term neonate gets, that is touch of his mother and is exposed to various noxious and aversive stimuli like heel prick handling by the NICU staff whose touch is unknown to them. Therefore Yakson touch might prove to have a soothing and calming effect on neonate behaviour, improve their sleep status and have a positive effect on social development of neonates ${ }^{2,8,24}$. Also kinaesthetic stimulation should be included in early intervention program to promote adaptive motor behaviour of neonates with $\mathrm{LBW}^{6,13}$.

Pederson et al examined the motor function of LBW preterm infants to predict developmental delays which may appear later and found that low birthweight infants exhibit different pattern of motor development in their first year of life as compared to full term infants ${ }^{24}$. Mahoney and Cohen in their review article concluded that there are evidences that early intervention assists the infants in age appropriate development ${ }^{7}$. Anderson stated that selected sensory input can facilitate physiological stability and growth if administered at an early age ${ }^{5}$. Therefore, early intervention is advocated to prevent the occurrence of neurodevelopmental disorders later in life. Individual studies have shown 
that Yakson touch is able to provide pain relief, improve sleep, affects their behavioural response, decreases the stress levels, increases maternal attachment, and has calming effects ${ }^{24,25}$. No adverse effects have been noted with Yakson ${ }^{25}$. Tactile kinaesthetic stimulation have been proven to have positive effects on the behaviour of neonates, such as motor development, self-regulated behaviour (regular breathing, state of alertness, balanced tone, a range of postures, coordinated movements, hand-to-face movement control, suction, grip, support, etc.), increased daily weight gain, adaptive behaviour and reduced hospital stay ${ }^{13,14}$. Medically unstable neonates, or neonates with disease or congenital anomaly are excluded as they will be already impoverished and for them life-saving interventions must be the priority; this too may allow uniformity in the intervention and results of those included. Also, this will avoid any interference in the treatments interventions being given to the already ailing neonate. Neonates with skin infection are excluded as direct touch might be a contraindication for them.

\section{References}

1. WHO | Preterm birth [Internet]. WHO. World Health Organization; 2016 [cited 2016 Dec 1]. Available from: http://www.who.int/topics/infant_newborn/en/

2. Bahman Bijari B, Iranmanesh S, Eshghi F, Baneshi MR. Gentle Human Touch and Yakson: The Effect on Preterm's Behavioral Reactions. ISRN Nurs 2012;2012(750363):1-6. doi:10.5402/2012/750363.

3. WHO | Infant, Newborn [Internet]. WHO. World Health Organization; 2016 [cited 2016 Dec 1]. Available from: http://www.who.int/mediacentre/factsheets/fs363/en/

4. Premature infant: MedlinePlus Medical Encyclopedia [Internet]. 2016. Available from: https://medlineplus. gov/ency/article/001562.htm

5. Anderson J. Sensory intervention with the preterm infant in the neonatal intensive care unit. Am J Occup Ther [Internet]. 1986 Jan;40(1):19-26. Available from: http://dx.doi.org/10.5014/ajot.40.1.19

6. Aliabadi F, Askary RK. Effects of Tactile - Kinesthetic Stimulation on Low Birth Weight Neonates. Iran J Pediatr [Internet]. 2013;23(3):289-94. Available from: http://www.ncbi.nlm.nih.gov/pmc/articles/ PMC3684473/pdf//JPD-23-289.pdf

7. Mahoney MC, Cohen MI. Effectiveness of developmental intervention in the neonatal intensive care unit: implications for neonatal physical therapy. Pediatr Phys Ther 2005;17(3):194-208.

8. Eshghi F, Iranmanesh S, Bahman Bijari B, Borhani F, Motamed Jahromi M. Effects of Yakson therapeutic touch on the behavioral response of premature infants. J Babol Univ Med Sci 2015;17(10):15-21.
The NBAS is chosen for assessment because it is reliable tool with the inter-rater reliability between 0.85 and $1.00^{21}$. It also incorporates all components of behaviour and motor development of the neonate. It is a tool for neurological and behavioural assessment capable of detecting any gross abnormality or asymmetry

To date there are limited studies on Yakson touch ${ }^{2,8,15}$. Therefore this study will aim to determine the combined effect of Yakson touch and Kinaesthetic stimulation on the behaviour of neonates. Should results be favourable it is anticipated that early intervention in the form of Yakson touch and kinaesthetic stimulation is added to the routine treatment of the high- risk neonates.

\section{Conclusion}

This protocol details a single centre trial to investigate if YAKIN may improve the behavioural responses of the high-risk neonates and kinaesthetic response may improve the motor behaviour of the highrisk of neonates.

9. Pepino VC, Aparecida M. Application of tactile / kinesthetic stimulation in preterm infants : a systematic review. J Pediatr (Rio J) Sociedade Brasileira de Pediatria; 2015;91(3):213-33. doi:10.1016/j. jped.2014.10.005.

10. Sweeney JK, Heriza CB, Blanchard Y. Neonatal physical therapy. Part I: Clinical competencies and neonatal intensive care unit clinical training models. Pediatr Phys Ther [Internet]. 2009;21(4):296-307. Available from: http://www.scopus.com/inward/record. url?eid=2-s2.0-74249091498\&partner|D $=40 \&$ md5 $=6$ 12aefa13901dcb3078d294cda8e0cab. doi:10.1097/ PEP.0b013e3181bf75ee.

11. Sweeney JK, Heriza CB, Blanchard Y, Dusing SC. Neonatal physical therapy. Part II: Practice frameworks and evidence-based practice guidelines. Pediatr Phys Ther 2010;22(1):2-16. doi:10.1097/ PEP.0b013e3181cdba43.

12. Neonatal Physical Therapy Practice: Roles and Training Recommendations for Developing Expertise in Neonatal Physical Therapy The Physical Therapist's Roles and Responsibilities in Neonatal Physical Therapy. [cited 2016 Dec 1]; Available from: www. pediatricapta.org

13. Askary R. Effect of Tactile-Kinesthetic Stimulation on Motor Development of Low Birth Weight Neonates. Iran Rehabil J [Internet]. 2011;9(13):16-8. Available from: http://irj.uswr.ac.ir/browse.php?a_id=201\&slc_ lang $=\mathrm{en} \& \mathrm{sid}=1 . \& \mathrm{ftxt}=1$

14. Ferreira AM, Bergamasco NHP. Behavioral analysis of preterm neonates included in a tactile and kinesthetic stimulation program during hospitalization. Rev Bras Fisioter. 2010;14(2):141-8. 
15. Parashar P, Samuel AJ, Bansal A, Aranha V. Yakson touch as a part of early intervention in the Neonatal Intensive Care Unit: A systematic narrative review. Indian J Crit Care Med [Internet]. 2016 Jun 13;20(6):349-52. Available from: http://www.ijccm. org/article. asp?issn=0972-5229. doi:10.4103/09725229.183897.

16. Portney LG, Watkins MP. Experimental Designs. In: Foundations of Clinical Research: Applications to Practice. 3rd ed. Philadelphia: FA Davis Company; 2015. p. 197-8.

17. Wang H, Chow S-C. Sample size calculation for comparing means. In: Wiley Encyclopedia of Clinical Trials [Internet]. John Wiley \& Sons, Inc.; 2007 [cited 2016 Dec 1]. p. 3-5. Available from: http://hansheng. gsm.pku.edu.cn/pdf/2007/mean.pdf

18. $\mathrm{WHO}$ | Kangaroo mother care: a practical guide. WHO [Internet]. World Health Organization; 2015 [cited 2016 Dec 14]; Available from: http://www.who.int/ maternal_child_adolescent/documents/9241590351/ en/

19. Hawthorne J. Using the Neonatal Behavioural Assessment Scale to support parent-infant relationships. Infant [Internet]. 2005;1(6):213-8. Available from: http://www.infantgrapevine.co.uk/pdf/ inf_006_irs.pdf

20. Noble Y, Boyd R. Neonatal assessments for the preterm infant up to 4months corrected age: A systematic review. Dev Med Child Neurol 2012;54(2):129-39.

21. Brazelton TB. Neonatal behavioral assessment scale. Clin Dev Med. 1973;(50):1-66.

22. Gupta SK. Intention-to-treat concept: A review. Perspect Clin Res [Internet]. India: Medknow Publications Pvt Ltd; 2011;2(3):109-12. Available from: http://www.ncbi.nlm.nih.gov/pmc/articles/ PMC3159210/. doi:10.4103/2229-3485.83221.

23. Soares I, Carneiro AV. Intention-to-treat analysis in clinical trials: principles and practical importance. Rev Port Cardiol 2002;21(10):1191-8.

24. Im H, Kim E, Cain KC. Acute effects of Yakson and Gentle Human Touch on the behavioral state of preterm infants. J Child Health Care 2009;13(3):21226. doi:10.1177/1367493509337441.

25. Park E-S, Sung K-S, Oh W-O, Im H-S, Kim E-S, Kim $\mathrm{Y}-\mathrm{A}$, et al. [Pain relieving effect of yakson therapy for infants]. Taehan Kanho Hakhoe Chi 2006;36(6):897904. 